\title{
When Are Pooled Panel-Data Regression Forecasts of Exchange Rates More Accurate than the Time-Series Regression Forecasts?
}

\author{
Nelson C. Mark \\ Donggyu Sul \\ University of Notre Dame and NBER University of Texas at Dallas
}

August 2011

\begin{abstract}
Out-of-sample forecasts of exchange rates in the late 1990s and 2000s generated by timeseries regression models have fared poorly. In mean-square error, these forecasts are typically dominated by the driftless random walk. On the other hand, pooled regression models estimated on panel data (and allowing for fixed effects) have, in many instances, performed much better than forecasts generated by time-series regression models. In this essay we investigate the conditions under which pooled regression forecasts generate more accurate exchange rate predictions than time-series regressions when the available econometric theory says that you should not pool. We offer an explanation that is based on finite sample considerations.
\end{abstract}




\section{Introduction}

Out-of-sample forecasts of exchange rates in the late 1990s and 2000s generated by time-series regression models have fared poorly. These forecasts are typically dominated (in mean-square error) by the driftless random walk. On the other hand, pooled regression models estimated on panel data (allowing for fixed effects) have, in many instances, performed much better than forecasts generated by time-series regression models. The superior predictive performance of the pooled panel data models is a puzzle because the evidence also tells of significant underlying model heterogeneity, in which case econometric theory tells us that pooling is inappropriate. Groen (2005), Rapach and Wohar (2004), Westerlund and Basher (2010) address the question about whether or not it is appropriate to pool. In this essay we ask when is it (under what conditions) that pooled regression forecasts generate more accurate exchange rate predictions than time-series regressions when the available econometric theory says that you should not pool.

The empirical literature upon which we focus traces its origin to, and is motivated toward overturning the findings of Meese and Rogoff (1983), who in studying floating exchange rates in the post Bretton Woods era, demonstrated that the driftless random walk model dominated economic theory based econometric models (e.g., purchasing-power parity models or simple monetary models) in out-of-sample forecast accuracy. Using time-series regression models, Mark (1995) and Chinn and Meese (1995) were able to overturn Meese and Rogoff by examination of long-horizon forecasts with error-correction models. The success of these papers was only temporary, however, because it was found that as time passed and more recent data from the 1990s and the 2000s were added to the time-series, the earlier findings of predictability became insignificant (Groen (1999), Cheung et al. (2005), Faust et al. (2003)). At the same time, other research (Lothian and Taylor (1996) and Rapach and Wohar (2002)) added observations to the front-end of the sample by constructing long historical time-series spanning over a hundred years or more. These studies found that simple monetary models and purchasing power parity based models have significant predictive ability. These papers are notable because their findings suggest that the inability to predict exchange rates with time-series regression models is a smallsample issue. The long time-span studies can be criticized, however, because they employed data that spanned across both fixed and flexible exchange rate regimes but they did not control for regime changes. The interpretation of the ability to predict out of sample based on a forecasting model estimated across different regimes is not entirely obvious.

One reaction to this state of affairs was to restrict attention to the post Bretton Woods float and to increase sample size in the cross-sectional dimension and exploiting panel data, 
as in Mark and Sul (2001), Groen (2005), Cerra and Saxena (2010), and Ince (2010). These studies found that forecasts built from pooled regression models estimated on panel-data dominate those of time-series regression forecasts as well as random walk forecasts in mean square. ${ }^{1}$ However, the data also tell us that there is significant heterogeneity across countries in the coefficients of the forecasting equation. Econometric theory instructs us in this case that one should not pool. But allowing for heterogeneous constants and slopes would generate little advantage over time-series regression, except to the extent that you could exploit correlated errors and do seemingly unrelated regression. Pooling when there is slope coefficient heterogeneity appears to commit, in the cross-sectional dimension, the same sort of error that failure to account for regime changes does in the time-series context.

The next section describes the methodology employed in the panel data exchange rate forecasting literature. In Section 3, we discuss the econometric theory that gives us some guidance in seeing the consequences from pooling when there is underlying heterogeneity. Section 4 contains a small Monte Carlo study that illustrates some of the ideas and predictions from the econometric theory. Section 5 is an application to data and Section 6 concludes.

\section{Panel data exchange rate determination studies}

In a data set of $N$ countries indexed by $i \in[1, N]$ and $T$ time-series observations indexed by $t \in[1, T]$, let $x_{i t}$ be a (scalar) prediction variable for $s_{i, t+k}$, the log exchange rate for country $i$. Mark and Sul (2001) and Cerra and Saxena (2010) investigate the empirical link between the monetary model fundamentals and the exchange rate. They set $x_{i, t}$ to be the deviation of today's exchange rate $s_{i, t}$ from the long-run equilibrium value predicted by economic theory. In the case of the monetary model, $x_{i, t}=f_{i, t}-s_{i, t}$, where $f_{i, t}=\left(m_{i, t}-m_{0, t}\right)-\lambda\left(y_{i, t}-y_{0, t}\right)$, country ' 0 ' is the base country, $m$ is the log money stock and $y$ is log real income. They used the panel exchange rate predictive regression

$$
s_{i, t+k}-s_{i t}=\beta x_{i t}+\eta_{i, t+k}
$$

where $\eta_{i, t+k}=\gamma_{i}+\theta_{t+k}+\epsilon_{i, t+k}$ has an error-components representation with individual (fixed) effect $\gamma_{i}$, common time effect $\theta_{t}$ and idiosyncratic effect $\epsilon_{i, t+k}$. Rapach and Wohar (2004) reject the null hypothesis of slope coefficient homogeneity at the one-percent level using Mark and Sul's data set.

\footnotetext{
${ }^{1}$ Groen (2000), Husted and MacDonald (1998) exploit panel data to test restrictions implied by the monetary model and find the evidence for the monetary model to be much stronger using panel data but these papers do not engage in out-of-sample prediction. Rogoff and Stavrakeva (2008) find that panel data models with time dummies do not produce robust forecasting results.
} 
Groen (2005) uses a four-country panel and pools a vector error correction model (VECM).

Letting $X_{i, t}=\left(s_{i, t},\left(m_{i, t}-m_{0, t}\right),\left(y_{i, t}-y_{0, t}\right)\right)^{\prime}$ and $Z_{i, t-1}=\left(X_{i, t-1}^{\prime}, 1\right)^{\prime}$, the model he works with is

$$
\Delta X_{i, t}=\alpha\left(1,-1, \phi, c_{i}\right) Z_{i, t-1}+\sum_{j=1}^{P} \Gamma_{i, j} \Delta X_{i, t-j}+\epsilon_{i, t},
$$

where $(1,-1, \phi)$ is the common cointegrating vector and $c_{i}$ is the regression constant. He finds that out-of-sample forecasts with the pooled VECM, beat the random walk and beat the unpooled (individual country) VECM forecasts.

In addition to forecasting studies, panel data has been used extensively in exchange rate research. Beginning with Frankel and Rose (1996), many studies have employed panel data to study long-run purchasing power parity (Papell (2006), Papell and Theodoridis (1998, 2001), Choi et al. (2006)), and cointegrating restrictions imposed by monetary models (Husted and MacDonald (1998), Groen (2000) and Rogoff and Stavrakeva (2008)). To the extent to which these papers pooled in the presence of cross-sectional heterogeneity, our comments may also have applicability. Engel, Mark and West (2010) employ factor models to predict the exchange rate. This is another use of panel data, but this sort of work is the topic of a separate paper.

\section{$3 \quad$ Asymptotic consequences of pooling}

Let $y_{i, t+k}=s_{i, t+k}-s_{i, t}$, where $s_{i, t}$ is the exchange rate between country $i$ and the base country, in logarithms. Suppose that economic theory suggests to us that $x_{i t}$ contains predictive information for future $s_{i, t}$. For example, we might think that the real exchange rate is mean reverting. In this case, we might let $x_{i t}$ be the deviation from purchasing power parity if we thought that the nominal exchange rate chases the relative price differential after a shock. Alternatively, we might let $x_{i t}$ be the deviation of $s_{i t}$ from a long-run specification of the equilibrium exchange rate. Simple monetary models suggest using some linear combination of the logarithm of country $i^{\prime} s$ money stocks, interest rates, and real GDP relative to those of the base country. Macroeconomic panel data sets typically have $T>N$. The existence of predictive ability has been investigated in two ways. The preferred method in the finance literature is to estimate a predictive regression for asset returns-that is a regression of future returns on currently observed data-and drawing inference on the slope coefficients using the full data set (Fama and French (1988), Daniel (2001), Stambaugh (1999)). The preferred method in research on exchange rates has been to employ out-of-sample prediction procedures and to examine the properties of the prediction errors. 


\subsection{Predictive regression estimated on full sample}

Suppose that the truth is

$$
y_{i, t+1}=\beta x_{i t}+\epsilon_{i, t+1},
$$

where $\epsilon_{t}=\left(\epsilon_{1, t}, \ldots, \epsilon_{N, t}\right) \stackrel{i i d}{\sim}\left(0, \sigma_{\epsilon}^{2}\right)$. This is the case where the slope is identical across countries. In practice, one always includes a (possibly heterogeneous) constant in the regression but we will ignore it in the exposition. This is a predictive regression in the sense that future changes in the exchange rate are projected onto current values of $x_{i t}$. We will assume that this is a wellbehaved regression. This will be true if $x_{i t}$ is econometrically exogenous. If this assumption seems too strong, one may assume that an appropriate bias correction method is applied. The asymptotic results that we discuss will not be affected if this is the case. The existence of predictive ability can be tested by estimating the predictive equation (1) in the full sample $(i=1, \ldots, N, t=1, \ldots, T)$ and doing a t-test of the null hypothesis $H_{0}: \beta=0$ against the alternative $H_{A}: \beta \neq 0$.

Asymptotic distribution theory tells us there are advantages to pooling if the truth is given by (1). An easy way to see this is by looking at the convergence rate of the pooled least-squares estimator,

$$
\sqrt{N T}(\hat{\beta}-\beta) \stackrel{d}{\rightarrow} N\left(0, \sigma_{\epsilon}^{2} Q^{-1}\right)
$$

where $Q=\operatorname{plim}\left((N T)^{-1} \sum_{i} \sum_{t} x_{i t}^{2}\right)$. Thus, the pooled estimator converges in distribution at the rate $\sqrt{N T}$ whereas the least-squares estimator for the time-series regression (not pooled) converges at rate $\sqrt{T}$. This suggests that asymptotically, there is an informational advantage to be gained by expanding the available observations in the cross-sectional dimension when the slopes are identical across $i$.

Now to compare to the case where there is underlying heterogeneity in the regression slopes, let the truth be

$$
\begin{gathered}
y_{i, t+1}=\beta_{i} x_{i, t}+\epsilon_{i, t+1} \\
\beta_{i} \stackrel{i i d}{\sim}\left(\beta, \sigma_{\beta}^{2}\right) .
\end{gathered}
$$

Eq. (4) characterizes the underlying heterogeneity of the slopes. Evidence of underlying heterogeneity in the slope coefficients across individuals has been reported by Groen (2005), Rapach and Wohar (2004), and Westerlund and Basher (2010). Pooling in the presence of heterogeneous slopes distorts the error term $u_{i, t+1}$ in

$$
\begin{aligned}
& y_{i, t+1}=\beta x_{i t}+u_{i, t+1}, \\
& u_{i, t+1}=\left(\beta_{i}-\beta\right) x_{i t}+\epsilon_{i, t+1} .
\end{aligned}
$$


As a result of this distortion, the asymptotic distribution of the pooled estimator is

$$
\sqrt{N T}(\hat{\beta}-\beta) \stackrel{d}{\rightarrow} N\left(0,\left(\omega^{2} Q^{-1}+\sigma_{\epsilon}^{2} Q^{-1}\right)\right)
$$

where $\omega^{2}$ and $\sigma_{\epsilon}^{2}$ are the asymptotic variances of $\left(\frac{1}{\sqrt{N T}} \sum_{i=1}^{N} \sum_{t=1}^{T}\left(\beta_{i}-\beta\right) x_{i t}^{2}\right)$ and $\left(\frac{1}{\sqrt{N T}} \sum_{i=1}^{N} \sum_{t=1}^{T} \epsilon_{i, t+1}\right)$ respectively.

To discuss the pros and cons of pooling, we compare the asymptotic distribution of the time-series regression (OLS) estimator with the pooled regression estimator. Under the null hypothesis of no predictability, $\left(\beta_{i}=\beta=0\right)$, let us rewrite the asymptotic distribution of the pooled estimator (5)) as

$$
\sqrt{T}(\hat{\beta}-0) \stackrel{d}{\rightarrow} N\left(0, \frac{\omega^{2} Q^{-1}+\sigma_{\epsilon}^{2} Q^{-1}}{N}\right) .
$$

The asymptotic distribution of the OLS estimator in the time-series case is of course,

$$
\sqrt{T}\left(\hat{\beta}_{i}-0\right) \stackrel{d}{\rightarrow} N\left(0, \sigma_{\epsilon}^{2} Q^{-1}\right) .
$$

Now comparing (6) and (7) we see that the asymptotic variance of the pooled regression can easily be smaller than that of the time-series regresssion estimator due to the $N$ in the denominator. This suggests that under the null hypothesis, the test of the null may be better sized than the pooled regression. This is only a suggestion, however, because in practice there may be many nuisance parameter issues such as cross sectional and serial dependencies that need to be dealt with and these complications can substantially distort the size of the test. These complications are assumed away here.

An alternative way to see potential advantages from pooling in the presence of slope heterogeneity is through an analysis of local alternative power. Let the sequence of local alternatives be given by

$$
\beta_{i}=\frac{c_{i}}{\sqrt{T}}, \quad \beta_{N}=\frac{b}{\sqrt{T}}
$$

where $b=\frac{1}{N} \sum_{i=1}^{N} c_{i}=O(1)$. Here we consider only the time $T$ dimension under the local alternative in order to compare the power of the test between by pooling and not pooling. Under these circumstances, the estimators in the time-series regression and the pooled regression have the (respective) asymptotic distributions,

$$
\begin{aligned}
& \frac{\sqrt{T}\left(\hat{\beta}_{i}-0\right)}{\sqrt{\sigma_{\epsilon}^{2} Q^{-1}}} \stackrel{d}{\rightarrow} N\left(\frac{c_{i}}{\sqrt{\sigma_{\epsilon}^{2} Q^{-1}}}, 1\right), \\
& \frac{\sqrt{N T}(\hat{\beta}-0)}{\sqrt{\Omega}} \stackrel{d}{\rightarrow} N\left(\frac{b}{\sqrt{\Omega}} N, 1\right) .
\end{aligned}
$$


where $\Omega=\omega^{2} Q^{-1}+\sigma_{\epsilon}^{2} Q^{-1}$. Hence the pooled test is potentially more powerful with even a moderately large $N$ because the local alternative parameter is $b N / \sqrt{\Omega}$. This means as $N$ increases, the alternative moves farther away from the null which increases the power of the test.

To summarize, if the goal is to test for predictive power, it might make sense to pool whether or not one believes that there is underlying heterogeneity in the slope coefficients. This is not to say that one can obtain more accurate forecasts using the pooled prediction equation, however. We are saying only that the test of the null hypothesis of no predictability can be easier to reject. There are a different set of issues involved in whether or not the pooled forecasts are actually more accurate. We now turn to an analysis of this topic.

\subsection{Out-of-sample prediction}

In the exchange rate literature, it is common to assess predictive ability with an analysis of out-of-sample forecasts. We let the full sample size in the time dimension be $T=S+P$. The model is estimated on the sample $t \in[1, S]$ and the observations $t \in[S+1, S+P]$ are reserved for assessing forecast accuracy. The asymptotic results discussed below assume that $S, P \rightarrow \infty$ so that the full sample size $S+P$ increases faster than the portion of the sample used for estimation. The standard practice is to generate the $P$ forecasts with recursively updated model estimates or with models estimated on a rolling sample. To simplify the exposition in this section, we assume that the model is estimated once on the first $S$ observations. However, the empirical work that follows below is done with rolling regressions. While doing so is a bit sloppy, it is innocuous in terms of the asymptotic theory because as $S \rightarrow \infty$, the recursive and rolling estimators have the same asymptotic distribution.

Again, let us assume that the truth is given by (1) in which there is no underlying heterogeneity in the slopes and one uses the sample $t \in[1, S]$ to estimate the predictive equation by pooled regression. Let $\hat{\beta}_{N S}$ and $\hat{\beta}_{S}$ be the pooled and time-series estimators of the slope and $\hat{y}_{i, j}^{\text {pool }}=\hat{\beta}_{N S} x_{i, j}, \hat{y}_{i, j}^{\text {time }}=\hat{\beta}_{S} x_{i, j}, j \in[S+1, S+P]$ be the predictors. Then as $N, S \rightarrow \infty$, the forecast errors have the representation

$$
\begin{aligned}
& \hat{y}_{i, j+1}^{\text {pool }}-y_{i, j+1}=\left(\hat{\beta}_{N S}-\beta\right) x_{i, j}+\epsilon_{i, j+1}=\epsilon_{i, j+1}+O_{p}\left(\frac{1}{\sqrt{N S}}\right) \\
& \hat{y}_{i, j+1}^{\text {time }}-y_{i, j+1}=\left(\hat{\beta}_{S}-\beta\right) x_{i, j}+\epsilon_{i, j+1}=\epsilon_{i, j+1}+O_{p}\left(\frac{1}{\sqrt{S}}\right)
\end{aligned}
$$

Hence, we have that the pooled forecast should always be more accurate than the time-series forecast in the finite sample. Asymptotically, however, makes no difference whether one pools 
or not because there is no difference in forecast accuracy between pooled and unpooled. ${ }^{2}$

Next, let us assume that the slope coefficients vary across countries and the truth is given by (3) and (4). The prediction errors from the pooled regression forecast are now

$$
\hat{y}_{i, j+1}^{\text {pool }}-y_{i, j+1}=\left(\hat{\beta}_{N S}-\beta_{i}\right) x_{i, j}+\epsilon_{i, j+1},
$$

whereas the time-series forecast errors are

$$
\hat{y}_{i, j+1}^{t i m e}-y_{i, j+1}=\left(\hat{\beta}_{i S}-\beta_{i}\right) x_{i, j}+\epsilon_{i, j+1} .
$$

Asymptotically,

$\operatorname{plim}_{N, S, P \rightarrow \infty} \frac{1}{P} \sum_{t=S+1}^{S+P}\left(\hat{y}_{i, j+1}^{\text {pool }}-y_{i, j+1}\right)^{2}=\sigma_{\epsilon}^{2}+\sigma_{x}^{2} \sigma_{\beta}^{2}>\sigma_{\epsilon}^{2}=\operatorname{plim}_{S, P \rightarrow \infty} \frac{1}{P} \sum_{t=S+1}^{S+P}\left(\hat{y}_{i, j+1}^{\text {time }}-y_{i, j+1}\right)^{2}$.

Asymptotically, therefore, pooling in the presence of underlying slope heterogeneity must lead to less accurate predictions asymptotically.

The question here is why do researchers often obtain lower mean-square prediction errors from pooled regression forecasts even when slope heterogeneity is present? At a general level, the reason is that guidance on whether to pool or not is based on asymptotic theory which may be inaccurate in any finite sample. To see why this may be the case, we get a clue by examining the difference in the squared prediction errors from the pooled and time-series regressions when there is underlying heterogeneity in the slopes. For any finite $S$ and $P$, as $N \rightarrow \infty$, this difference is

$$
\left(\hat{y}_{i, j+1}^{\text {pool }}-y_{i, j+1}\right)^{2}-\left(\hat{y}_{i, j+1}^{\text {time }}-y_{i, j+1}\right)^{2}=\left[\left(\hat{\beta}_{N S}-\beta_{i}\right)^{2}-\left(\hat{\beta}_{i S}-\beta_{i}\right)^{2}\right] x_{i, j}^{2}+o_{p}(1) .
$$

Thus, in any finite sample, it is possible for $\left(\hat{\beta}_{N S}-\beta_{i}\right)^{2}<\left(\hat{\beta}_{i S}-\beta_{i}\right)^{2}$, in which case the pooled predictor will perform better. This is most likely the case when the underlying dispersion in the slope coefficients is not too large.

Probing deeper, however, we can see that for each $i$, the relative forecasting performance depends on two parts: the distance of the pooled estimates $\hat{\beta}_{N S}$ from the unknown true value of $\beta_{i}$, and the distance between the time series estimates $\hat{\beta}_{i S}$ and the true value. In practice, with a large $N$ but relatively small $S$ the pooled estimates are robust and do not move around

\footnotetext{
${ }^{2}$ As $S \rightarrow \infty$ regardless size of $N$, $\operatorname{plim}_{S, P \rightarrow \infty} \frac{1}{P} \sum_{t=S+1}^{S+P}\left(\hat{y}_{i, j+1}^{\text {pool }}-y_{i, j+1}\right)^{2}=\operatorname{plim}_{S, P \rightarrow \infty} \frac{1}{P} \sum_{t=S+1}^{S+P}\left(\hat{y}_{i, j+1}^{\text {time }}-y_{i, j+1}\right)^{2}=\sigma_{\epsilon}^{2}$.
} 
much as the time-series estimates as the length of the sample changes since its variance of $\hat{\beta}_{N S}$ is $O_{p}\left(N^{-1} S^{-1}\right)$. Meanwhile the time series estimates do tend to move around as $S$ increases because the variance of $\hat{\beta}_{i S}$ is $O_{p}\left(S^{-1}\right)$. Now if the pooled estimate is sufficiently stable so that we can treat it as if it is constant, then the relative forecasting performance will depend primarily on the variability of the time series estimator. In the next section, we explore these ideas further with a small Monte Carlo study.

\section{Monte Carlo Study}

In our data generating process (DGP), the cross-sectional dimension is $N=10$, and the timeseries dimension varies from $S+P=100$ to 400 . For each time-series length, estimation is performed over half of the sample and prediction over the second half so that $S=P$. For example, the smallest sample size that we consider is of length 100. The first 50 observations are used to estimate the prediction model and the second 50 are reserved for forecast evaluation.

The data generating process (DGP) for our Monte Carlo work is as follows:

$$
\begin{aligned}
y_{i, t+1} & =\beta_{i} x_{i t}+\epsilon_{i, t+1}, \\
x_{i t} & \stackrel{i i d}{\sim} N(0,1), \\
\epsilon_{i t} & \stackrel{i i d}{\sim} N(0,1) .
\end{aligned}
$$

In terms of the slope heterogeneity, we consider the following three cases considered for assignment of the slope coefficients:

\begin{tabular}{cccc}
\hline \multicolumn{4}{c}{$\beta_{i}$ assignment } \\
\hline Cross-sectional unit & Case 1 & Case 2 & Case 3 \\
\hline 1 & 0.05 & 0.05 & 0.05 \\
2 & 0.06 & 0.10 & 0.15 \\
3 & 0.07 & 0.15 & 0.25 \\
4 & 0.08 & 0.20 & 0.35 \\
5 & 0.09 & 0.25 & 0.45 \\
6 & 0.10 & 0.30 & 0.55 \\
7 & 0.11 & 0.35 & 0.65 \\
8 & 0.12 & 0.40 & 0.75 \\
9 & 0.13 & 0.45 & 0.85 \\
10 & 0.14 & 0.50 & 0.95 \\
\hline
\end{tabular}


For each experiment, 2000 replications are performed. We compute the mean square prediction error from the pooled regression forecasts, the time-series regression forecast, and the driftless random walk forecast. Our discussion is limited to these point estimates. Inference issues, while very important, are addressed in a separate chapter of this volume (Corte and Tsiakas (2010)). ${ }^{3}$

Table 1 shows the mean-square prediction error (MSPE) comparison between the pooled and time-series forecasts. Bold entries indicate instances in which the MSPE of the pooled forecasts dominate those of the time-series forecasts. We observe two very striking features, which corroborate the implications of the asymptotic analysis in the previous section. The pooled forecasts tend to dominate the time-series forecasts when (i) the sample size is small in the time dimension and (ii) when the slope heterogeneity across individuals is not too large (i.e., case 1 as opposed to case 3 ).

Table 2 reports the MSPE of the time-series regression forecasts relative to the driftless random walk. Beginning with the work of Meese and Rogoff (1983), the random walk has been the standard benchmark against which econometric forecasts are judged in the empirical exchange rate literature. The message from Table 2 is that the alternative has to be fairly far away from the null ( $\beta_{i}$ has to be fairly sizable) before predictions from the time-series regression model can beat the random walk, even in sample sizes as large as 200. Typically, in exchange rate research, we have access to short time series and slope coefficient estimates tend to be small in magnitude.

In Table 3, the MSPE of the pooled forecasts are compared to those of the random walk. Again, the pooled forecast will generally perform well relative to the random walk when the underlying model heterogeneity is small and when the time series is short. Notice case 3 . When there is substantial heterogeneity in the slope coefficients, the pooled forecasts dominate the random walk in cases far from the null (large $\beta_{i}$ ) and are beaten badly when the $\beta_{i}$ are small.

\section{$5 \quad$ An illustration with data}

In this section, we illustrate these ideas using data for US dollar exchange rates against the currencies of Australia, Canada, Chile, Colombia, the Czech Republic, Denmark, Hungary, Israel, South Korea, Norway, New Zealand, the Phillipines, the Russian Federation, Singapore,

\footnotetext{
${ }^{3}$ The iid assumption for $x_{i t}$ is innocuous because the relative forecasting performance expressed in ratios does not depend on $x_{i, j}^{2}$. With a highly serially correlated regressor the asymptotic results don't change but in finite samples, the relative dispersion of $\hat{\beta}_{i T}$ to $\hat{\beta}_{N T}$ may increase with the degrees of serial correlation. If this is the case, the Monte Carlo results may show overly favorable results for the pooled forecasts.
} 
South Africa, Sweden, Taiwan, the United Kingdom, Japan, Switzerland, and the Euro Area. The dimensions of the data set are $N=21, S+P=132$. We are using monthly observations that extend from January 1999 through January 2010 obtained from Global Insights. ${ }^{4}$ We use observations from January 1999 through December 2003 initially to estimate the prediction model and to form forecasts at horizons of 1 to 24 months ahead. We then recursively update the sample and repeat the estimation and forecast generation. Relative forecast accuracy is measured by Theil's U-Statistic, which is the ratio of the RMPSE of a particular model to that of the driftless random walk.

The predictive variable is based on purchasing power parity. For country $i$, we set

$$
x_{i, t}=\left(p_{i t}-p_{0, t}\right)-s_{i, t},
$$

where $p$ is the log price level and the ' 0 ' subscript refers to the US. For the time-series regression, the $k$-month ahead predictive equation is

$$
s_{i, t+k}-s_{i, t}=\alpha_{i}+\beta_{i} x_{i, t}+\epsilon_{i, t+k} .
$$

For the pooled regression case with fixed-effects, the predictive equation is

$$
s_{i, t+k}-s_{i, t}=\alpha_{i}+\beta x_{i, t}+\epsilon_{i, t+k}
$$

This is the model of Mark and Sul (2001) but without controls (time-specific dummy variables) for a common time effect. The regression errors are in fact correlated across $i$ and if we were concerned with drawing inference, then it would be necessary to model the cross-sectional dependence in the regression error. However, since we are reporting only the point estimates, we can safely ignore this complication.

The relative prediction results are shown in Table 4. The Table shows the ratio of Theil's $\mathrm{U}$ statistic for the pooled forecast to that of the time-series forecast. We generate forecasts at horizons 1 through 24 but we report only detailed results for horizons 1 through 6 . The last column shows the average U-statistic ratio over forecast horizons 1 through 24 . Values less than 1 indicate that the pooled forecast is more accurate in the mean-square sense. The pooled forecast is more accurate than the time-series forecast in about half of the cases. As can be seen from the last column, the dominance of the pooled forecasts becomes stronger at the longer horizons.

To help identify when it is that the pooled forecast will dominate the time-series forecast, we work with eq.(10). First, we note that the relative ranking of the mean square error for

\footnotetext{
${ }^{4}$ For New Zealand and Australia, quarterly CPI is interpolated into monthly. The fundamentals for the euro are an aggregation of variables in the euro zone.
} 
currency $i$ depends on the distance between the particular estimator (pooled or unpooled) and the true slope value $\beta_{i}$,

$$
\begin{aligned}
& \operatorname{sign}\left\{\frac{1}{P} \sum_{t=S+1}^{S+P}\left(\hat{y}_{i, j+1}^{\text {pool }}-y_{i, j+1}\right)^{2}-\frac{1}{P} \sum_{t=S+1}^{S+P}\left(\hat{y}_{i, j+1}^{\text {time }}-y_{i, j+1}\right)^{2}\right\}, \\
& =\operatorname{sign}\left\{\frac{1}{P} \sum_{p=1}^{P}\left\{\left(\hat{\beta}_{N S+p}-\beta_{i}\right)^{2}-\left(\hat{\beta}_{i S+p}-\beta_{i}\right)^{2}\right\}\right\} .
\end{aligned}
$$

Let us call the last expression, $\gamma_{P, i} \equiv \frac{1}{P} \sum_{p=1}^{P}\left\{\left(\hat{\beta}_{N S+p}-\beta_{i}\right)^{2}-\left(\hat{\beta}_{i S+p}-\beta_{i}\right)^{2}\right\}$. Then the pooled forecast will dominate the time-series forecast when $\gamma_{P, i}<0$. To verify this prediction, we compute the average $\gamma_{P, i}$ for one-to-six period ahead forecasts and plot them against the (average of ) the relative Theil's U-statistic in Figure 1. ${ }^{5}$ The results conform to the predictions of the theory in that the $\gamma_{P, i}$ are positively correlated with the relative Theil's U-statistics, and the pooled forecasts tend to dominate the time-series forecasts for those $i$ in which $\gamma_{P, i}<$ 0 . The figure predicts that the pooled forecasts will dominate the time-series forecasts for Columbia, the Philippines, Taiwan, Russia, Singapore, Hungary, Denmark, and the Euro zone, and referring back to Table 6 confirms this prediction. Predictions of these exchange rates should be generated by the pooled panel data model. However, currencies of Sweden, the UK, Japan, and Israel should employ time-series regression.

\section{Conclusions}

The empirical literature finds that out-of-sample exchange rate forecasts over the 1990s and 2000s from (pooled) panel data models often perform better than those generated from timeseries regression models. Researchers have reported these findings, even though there is substantial model heterogeneity across countries (or currencies) and asymptotic analysis tells us

$$
\begin{aligned}
& { }^{5} \text { In calculating } \gamma_{P, i} \text {, we note that the difference in the mean square errors can be approximated as } \\
& \begin{aligned}
\gamma_{P, i} & =\frac{1}{P} \sum_{p=1}^{P}\left\{\left(\hat{\beta}_{N S+p}-\hat{\beta}_{S+P, i}\right)^{2}-\left(\hat{\beta}_{i S+p}-\hat{\beta}_{S+P, i}\right)^{2}\right\} \\
& =\frac{1}{P} \sum_{p=1}^{P}\left(\hat{\beta}_{N S+p}-\beta_{i}\right)^{2}-\frac{1}{P} \sum_{p=1}^{P}\left(\hat{\beta}_{i S+p}-\beta_{i}\right)^{2}-2 \frac{1}{P} \sum_{p=1}^{P}\left(\hat{\beta}_{N S+p}-\hat{\beta}_{i S+p}\right)\left(\beta_{i}-\hat{\beta}_{S+P, i}\right) \\
& =\frac{1}{P} \sum_{p=1}^{P}\left(\hat{\beta}_{N S+p}-\beta_{i}\right)^{2}-\frac{1}{P} \sum_{p=1}^{P}\left(\hat{\beta}_{i S+p}-\beta_{i}\right)^{2}+O_{p}\left(\frac{1}{\sqrt{S+P}}\right) .
\end{aligned}
\end{aligned}
$$

as $P \rightarrow \infty$. The last step follows since $\left(\beta_{i}-\hat{\beta}_{S+P, i}\right)=O_{p}\left(\frac{1}{\sqrt{S+P}}\right)$ but $\left(\hat{\beta}_{N S+p}-\hat{\beta}_{i S+p}\right)=O_{p}(1)$. Hence, we approximate the unobserved true value $\beta_{i}$ with the point estimate using the full sample, $\hat{\beta}_{S+P, i}$. 
that pooling should be inappropriate in this case.

What we have shown is that if one is interested in evaluating out-of-sample forecast ability, pooling does not always generate more accurate forecasts than time-series regression. Pooling dominates when there is not much heterogeneity in the model parameters. When the heterogeneity is great, time-series forecasts should be used. However, if one is interested in testing for predictive ability using the full sample, pooling will result in more powerful tests as long as the model parameters and regressors are uncorrelated with the regression errors. Under these conditions, it makes sense to pool whether or not one believes there is underlying slope heterogeneity. 


\section{References}

[1] Campbell, John Y. and Robert J. Shiller, (1988), 'Stock Prices, Earnings, and Expected Dividends,' Journal of Finance, July, 43, pp. 661-76.

[2] Cerra, Valerie and Sweta Chaman Saxena, (2010). "The monetary model strikes back: Evidence from the world," Journal of International Economics, 81:184196.

[3] Cheung, Yin-Wong, Menzie D. Chinn and Antonio Garcia Pascual, (2005). "Empirical exchange rate models of the nineties: Are any fit to survive?" Journal of International Money and Finance, 24: 1150-1175.

[4] Chinn, M.D., Meese, R.A., (1995). "Banking on currency forecasts: how predictable is change in money?" Journal of International Economics 38, 161-178.

[5] Choi, Chi-Young, Nelson C. Mark and Donggyu Sul, (2006). "Unbiased Estimation of the Half-Life to PPP Convergence in Panel Data," Journal ofMoney, Credit, and Banking, 38: 921—938.

[6] Corte, Pasquale Della and Ilias Tsiakas, (2010). "Statistical and Economic Methods for Evaluating Exchange Rate Predictability," mimeo, University of Warwick.

[7] Daniel, Kent, 2001. "The Power and Size of Mean Reversion Tests," Journal of Empirical Finance, 8, 493-535.

[8] Engel, Charles, Nelson C. Mark and Kenneth D. West, (2009). "Factor Model Forecasts of Exchange Rates," mimeo, University of Wisconsin.

[9] Fama, Eugene F. and Kenneth R. French, (1988). 'Dividend Yields and Expected Stock Returns,' Journal of Financial Economics, October, 22, pp.3-25.

[10] Faust, J., Rogers, J.H., Wright, J., (2003). "Exchange rate forecasting: the errors we've really made." Journal of International Economics 60, 35-59.

[11] Frankel, Jeffrey A., and Andrew K. Rose (1996). "A Panel Project on Purchasing Power Parity: Mean Reversion within and between Countries." Journal of International Economics 40, 209-224.

[12] Goetzmann, William N. and Philippe Jorion, (1993). "Testing the Predictive Power of Dividend Yields," Journal of Finance, 48, 663-79.

[13] Groen, Jan J. J. (1999). "Long horizon predictability of exchange rates: Is it for real?" Empirical Economics, 24: 451-469. 
[14] Groen, Jan J.J. (2000). "The monetary exchange rate model as a long-run phenomenon," Journal of International Economics, 52: 299-319.

[15] Groen, Jan J.J. (2005). "Exchange Rate Predictability and Monetary Fundamentals in a Small Multi-Country Panel," Journal of Money, Credit, and Banking, 37(3): 495-516.

[16] Husted, Steven and Ronald Macdonald, (1998). "Monetary-based models of the exchange rate: a panel perspective," Journal of International Financial Markets, Institutions and Money, 8: 1-19.

[17] Ince, Onur, (2010). "Forecasting Exchange Rates Out-of-Sample with Panel Methods and Real-Time Data", mimeo, University of Houston.

[18] Lothian, James R. and Mark P. Taylor, (1996). "Real exchange rate behavior: the recent float from the perspective of the past two centuries," Journal of Political Economy, 104: 488-510.

[19] McCraken, Michael W. and Stephen Graham Sapp, (2005). "Evaluating the Predictability of Exchange Rates Using Long Horizon Regressions: Mind Your p's and q's!" Journal of Money, Credit, and Banking, 37(3): 473-494.

[20] Meese, Richard and Kenneth Rogoff, (1983). "Empirical Exchange Rate Models of the 1980's: Do They Fit Out of Sample?" Journal of International Economics, 14: 3-24.

[21] Mark, Nelson C. (1995). "Exchange Rates and Fundamentals: Evidence on LongHorizon Predictability," American Economic Review, 85: 201-218.

[22] Mark, Nelson C. and Donggyu Sul, (2001). "Nominal Exchange Rates and Monetary Fundamentals: Evidence from a Small Post-Bretton Woods Panel," Journal of International Economics, 53: 29-52

[23] Papell, David H. (2006). "The Panel Purchasing Power Parity Puzzle." Manuscript, Journal of Money, Credit, and Banking, 447-467)

[24] Papell, David H., and Hristos Theodoridis (1998). "Increasing Evidence of Purchasing Power Parity over the Current Float." Journal of International Money and Finance 17, 41-50.

[25] Papell, David H., and Hristos Theodoridis (2001). "The Choice of Numeraire Currency in Panel Tests of Purchasing Power Parity." Journal of Money, Credit, and Banking 33, 790-803. 
[26] Rapach, David E. and Mark E. Wohar, (2002). "Testing the monetary model of exchange rate determination: new evidence from a century of data," Journal of International Economics, 58: 359-385.

[27] Rapach, David E. and Mark E. Wohar, (2004). "Testing the monetary model of exchange rate determination: a closer look at panels," Journal of International Money and Finance, 23: 867-895.

[28] Rogoff, Kenneth and Vania Stavrakeva, (2008). "The Continuing Puzzle of Short Horizon Exchange Rate Forecasting," mimeo, Harvard University.

[29] Stambaugh, Robert F. 1999. "Predictive Regressions," Journal of Financial Economics, 54, 375-421.

[30] Richardson, Matthew and James Stock, 1989. "Drawing Inferences from Statistics Based on Multiyear Asset Returns," Journal of Financial Economics, 25, 34348.

[31] Westerlund, Joakim and Syed A. Basher, (2007). "Can Panel Data Really Improve the Predictability of the Monetary Exchange Rate Model?" Journal of Forecasting, 26: 365-383. 
Figure 1. Average of Theil's U of pooled forecast relative to time-series forecast over horizons 1 through 6 plotted against average $\gamma_{P, i}$.

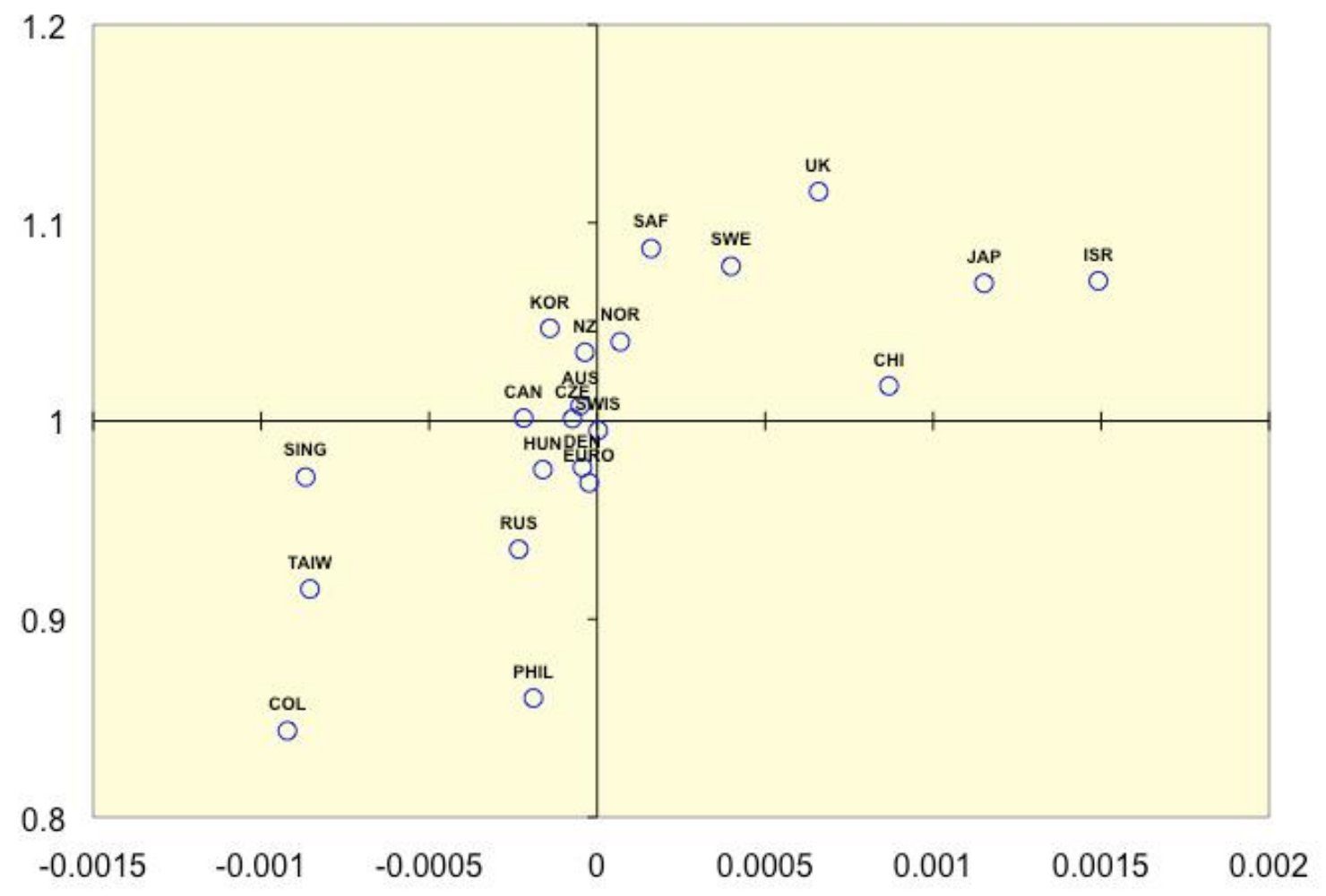


Table 1: $\mathrm{MSPE}_{\text {pool }} / \mathrm{MSPE}_{\text {time }}$

\begin{tabular}{|c|c|c|c|c|c|c|c|c|c|c|}
\hline$P=S$ & $i=1$ & $i=2$ & $i=3$ & $i=4$ & $i=5$ & $i=6$ & $i=7$ & $i=8$ & $i=0$ & $i=10$ \\
\hline & \multicolumn{10}{|c|}{ Case $1: \beta=(0.05,0.06, \ldots, 0.14)$} \\
\hline 50 & 0.990 & 0.989 & 0.989 & 0.987 & 0.987 & 0.988 & 0.989 & 0.988 & 0.990 & 0.989 \\
\hline 60 & 0.992 & 0.990 & 0.991 & 0.990 & 0.989 & 0.990 & 0.990 & 0.990 & 0.991 & 0.992 \\
\hline 70 & 0.993 & 0.993 & 0.993 & 0.991 & 0.991 & 0.992 & 0.991 & 0.992 & 0.993 & 0.993 \\
\hline 100 & 0.996 & 0.995 & 0.994 & 0.994 & 0.994 & 0.994 & 0.994 & 0.994 & 0.995 & 0.995 \\
\hline \multirow[t]{2}{*}{200} & 0.999 & 0.998 & 0.997 & 0.997 & 0.997 & 0.997 & 0.997 & 0.997 & 0.998 & 0.999 \\
\hline & \multicolumn{10}{|c|}{ Case $2: \beta=(0.05,0.10, \ldots, 0.50)$} \\
\hline 50 & 1.041 & 1.018 & 1.003 & 0.994 & 0.988 & 0.989 & 0.993 & 1.003 & 1.020 & 1.040 \\
\hline 60 & 1.044 & 1.021 & 1.005 & 0.994 & 0.991 & 0.990 & 0.996 & 1.006 & 1.022 & 1.043 \\
\hline 70 & 1.041 & 1.024 & 1.006 & 0.998 & 0.992 & 0.992 & 0.997 & 1.008 & 1.022 & 1.044 \\
\hline 100 & 1.046 & 1.025 & 1.010 & 1.000 & 0.994 & 0.995 & 1.000 & 1.010 & 1.026 & 1.045 \\
\hline \multirow[t]{2}{*}{200} & 1.047 & 1.028 & 1.012 & 1.002 & 0.998 & 0.998 & 1.003 & 1.012 & 1.028 & 1.049 \\
\hline & \multicolumn{10}{|c|}{ Case $3: \beta=(0.05,0.15, \ldots, 0.95)$} \\
\hline 50 & 1.199 & 1.115 & 1.053 & 1.009 & 0.991 & 0.990 & 1.009 & 1.050 & 1.114 & 1.193 \\
\hline 60 & 1.192 & 1.116 & 1.053 & 1.014 & 0.993 & 0.993 & 1.011 & 1.056 & 1.116 & 1.195 \\
\hline 70 & 1.195 & 1.112 & 1.055 & 1.014 & 0.994 & 0.994 & 1.013 & 1.056 & 1.116 & 1.198 \\
\hline 100 & 1.201 & 1.117 & 1.057 & 1.017 & 0.996 & 0.997 & 1.017 & 1.056 & 1.116 & 1.200 \\
\hline 200 & 1.201 & 1.119 & 1.060 & 1.020 & 0.999 & 1.000 & 1.021 & 1.061 & 1.120 & 1.200 \\
\hline
\end{tabular}


Table 2: $\mathrm{MSPE}_{\text {time }} / \mathrm{MSPE}_{\text {r.w }}$

\begin{tabular}{|c|c|c|c|c|c|c|c|c|c|c|}
\hline$P=S$ & $i=1$ & $i=2$ & $i=3$ & $i=4$ & $i=5$ & $i=6$ & $i=7$ & $i=8$ & $i=9$ & $i=10$ \\
\hline & \multicolumn{10}{|c|}{ Case $1: \beta=(0.05,0.06, \ldots, 0.14)$} \\
\hline 50 & 1.012 & 1.011 & 1.010 & 1.009 & 1.007 & 1.005 & 1.001 & 1.001 & 0.999 & 0.997 \\
\hline 60 & 1.010 & 1.010 & 1.009 & 1.004 & 1.005 & 1.002 & 1.000 & 0.999 & 0.996 & 0.992 \\
\hline 70 & 1.008 & 1.008 & 1.005 & 1.004 & 1.004 & 1.000 & 0.998 & 0.996 & 0.993 & 0.992 \\
\hline 100 & 1.005 & 1.004 & 1.002 & 1.000 & 1.000 & 0.998 & 0.995 & 0.993 & 0.992 & 0.989 \\
\hline \multirow[t]{2}{*}{200} & 1.001 & 1.000 & 0.999 & 0.997 & 0.996 & 0.994 & 0.992 & 0.989 & 0.988 & 0.984 \\
\hline & \multicolumn{10}{|c|}{ Case $2: \beta=(0.05,0.10, \ldots, 0.50)$} \\
\hline 50 & 1.012 & 1.006 & 0.994 & 0.977 & 0.958 & 0.932 & 0.908 & 0.879 & 0.848 & 0.817 \\
\hline 60 & 1.009 & 1.003 & 0.991 & 0.972 & 0.957 & 0.931 & 0.903 & 0.877 & 0.844 & 0.814 \\
\hline 70 & 1.008 & 1.001 & 0.987 & 0.973 & 0.954 & 0.930 & 0.903 & 0.873 & 0.845 & 0.812 \\
\hline 100 & 1.005 & 0.998 & 0.985 & 0.970 & 0.949 & 0.925 & 0.897 & 0.870 & 0.838 & 0.808 \\
\hline \multirow[t]{2}{*}{200} & 1.001 & 0.994 & 0.981 & 0.965 & 0.948 & 0.920 & 0.893 & 0.867 & 0.835 & 0.802 \\
\hline & \multicolumn{10}{|c|}{ Case 3: $\beta=(0.05,0.15, \ldots, 0.95)$} \\
\hline 50 & 1.012 & 0.992 & 0.957 & 0.906 & 0.852 & 0.787 & 0.726 & 0.660 & 0.597 & 0.545 \\
\hline 60 & 1.009 & 0.991 & 0.953 & 0.906 & 0.850 & 0.784 & 0.724 & 0.652 & 0.595 & 0.543 \\
\hline 70 & 1.007 & 0.988 & 0.951 & 0.901 & 0.845 & 0.781 & 0.719 & 0.651 & 0.594 & 0.538 \\
\hline 100 & 1.004 & 0.986 & 0.948 & 0.898 & 0.841 & 0.775 & 0.711 & 0.651 & 0.589 & 0.534 \\
\hline 200 & 1.001 & 0.982 & 0.945 & 0.895 & 0.835 & 0.771 & 0.706 & 0.643 & 0.586 & 0.530 \\
\hline
\end{tabular}


Table 3: $\mathrm{MSPE}_{\text {pool }} / \mathrm{MSPE}_{r . w}$

\begin{tabular}{|c|c|c|c|c|c|c|c|c|c|c|}
\hline$P=S$ & $i=1$ & $i=2$ & $i=3$ & $i=4$ & $i=5$ & $i=6$ & $i=7$ & $i=8$ & $i=9$ & $i=10$ \\
\hline & \multicolumn{10}{|c|}{ Case $1: \beta=(0.05,0.06, \ldots, 0.14)$} \\
\hline 50 & 1.001 & 0.999 & 0.997 & 0.996 & 0.993 & 0.992 & 0.988 & 0.988 & 0.987 & 0.985 \\
\hline 60 & 1.001 & 0.999 & 1.000 & 0.994 & 0.993 & 0.991 & 0.990 & 0.988 & 0.986 & 0.984 \\
\hline 70 & 1.001 & 1.000 & 0.997 & 0.995 & 0.994 & 0.991 & 0.989 & 0.987 & 0.986 & 0.984 \\
\hline 100 & 1.001 & 0.999 & 0.996 & 0.995 & 0.993 & 0.991 & 0.989 & 0.987 & 0.986 & 0.984 \\
\hline \multirow[t]{2}{*}{200} & 1.000 & 0.998 & 0.996 & 0.994 & 0.992 & 0.991 & 0.989 & 0.987 & 0.985 & 0.983 \\
\hline & \multicolumn{10}{|c|}{ Case $2: \beta=(0.05,0.10, \ldots, 0.50)$} \\
\hline 50 & 1.053 & 1.024 & 0.998 & 0.971 & 0.946 & 0.920 & 0.900 & 0.878 & 0.860 & 0.843 \\
\hline 60 & 1.054 & 1.025 & 0.996 & 0.966 & 0.948 & 0.921 & 0.897 & 0.879 & 0.859 & 0.844 \\
\hline 70 & 1.050 & 1.025 & 0.994 & 0.972 & 0.947 & 0.922 & 0.899 & 0.877 & 0.860 & 0.843 \\
\hline 100 & 1.051 & 1.023 & 0.996 & 0.970 & 0.944 & 0.919 & 0.896 & 0.877 & 0.857 & 0.842 \\
\hline \multirow[t]{2}{*}{200} & 1.048 & 1.022 & 0.994 & 0.968 & 0.946 & 0.918 & 0.895 & 0.877 & 0.858 & 0.840 \\
\hline & \multicolumn{10}{|c|}{ Case $3: \beta=(0.05,0.15, \ldots, 0.95)$} \\
\hline 50 & 1.215 & 1.109 & 1.012 & 0.917 & 0.844 & 0.778 & 0.728 & 0.686 & 0.655 & 0.637 \\
\hline 60 & 1.204 & 1.108 & 1.007 & 0.921 & 0.845 & 0.777 & 0.728 & 0.683 & 0.656 & 0.637 \\
\hline 70 & 1.204 & 1.100 & 1.006 & 0.915 & 0.840 & 0.775 & 0.726 & 0.682 & 0.655 & 0.635 \\
\hline 100 & 1.207 & 1.103 & 1.004 & 0.914 & 0.838 & 0.771 & 0.721 & 0.684 & 0.653 & 0.634 \\
\hline 200 & 1.203 & 1.100 & 1.003 & 0.914 & 0.835 & 0.770 & 0.719 & 0.681 & 0.654 & 0.633 \\
\hline
\end{tabular}


Table 4: Theil's U-Statistics: Pooled/Time Series

\begin{tabular}{lcccccc|c}
\hline \hline Horizon & 1 & 2 & 3 & 4 & 5 & 6 & Avg over 24 \\
\hline Australia & $\mathbf{0 . 9 9 4}$ & $\mathbf{0 . 9 8 9}$ & $\mathbf{0 . 9 9 6}$ & 1.007 & 1.020 & 1.041 & $\mathbf{0 . 8 4 1}$ \\
Canada & $\mathbf{0 . 9 5 8}$ & $\mathbf{0 . 9 7 5}$ & 1.009 & 1.010 & 1.014 & 1.042 & $\mathbf{0 . 8 9 9}$ \\
Chile & $\mathbf{0 . 9 5 5}$ & $\mathbf{0 . 9 9 1}$ & 1.014 & 1.022 & 1.043 & 1.082 & 1.083 \\
Colombia & $\mathbf{0 . 9 2 7}$ & $\mathbf{0 . 8 9 3}$ & $\mathbf{0 . 8 4 4}$ & $\mathbf{0 . 8 0 9}$ & $\mathbf{0 . 7 9 7}$ & $\mathbf{0 . 7 9 2}$ & $\mathbf{0 . 7 4 0}$ \\
Czech & $\mathbf{0 . 9 8 7}$ & $\mathbf{0 . 9 8 3}$ & $\mathbf{0 . 9 9 2}$ & 1.009 & 1.022 & 1.015 & 1.325 \\
Denmark & $\mathbf{0 . 9 7 4}$ & $\mathbf{0 . 9 7 9}$ & $\mathbf{0 . 9 6 0}$ & $\mathbf{0 . 9 7 3}$ & $\mathbf{0 . 9 8 2}$ & $\mathbf{0 . 9 9 1}$ & $\mathbf{0 . 7 7 9}$ \\
Hungary & $\mathbf{0 . 9 8 2}$ & $\mathbf{0 . 9 8 1}$ & $\mathbf{0 . 9 8 0}$ & $\mathbf{0 . 9 7 2}$ & $\mathbf{0 . 9 7 2}$ & $\mathbf{0 . 9 6 7}$ & 1.084 \\
Israel & 1.020 & 1.012 & 1.027 & 1.069 & 1.121 & 1.176 & 1.317 \\
Korea & 1.001 & 1.012 & 1.024 & 1.047 & 1.083 & 1.113 & 1.234 \\
Norway & 1.011 & 1.011 & 1.024 & 1.050 & 1.069 & 1.074 & $\mathbf{0 . 9 6 7}$ \\
NZ & $\mathbf{0 . 9 9 0}$ & 1.010 & 1.031 & 1.050 & 1.058 & 1.069 & $\mathbf{0 . 9 6 4}$ \\
Phillipines & $\mathbf{0 . 9 6 0}$ & $\mathbf{0 . 9 2 6}$ & $\mathbf{0 . 8 8 3}$ & $\mathbf{0 . 8 3 7}$ & $\mathbf{0 . 8 0 9}$ & $\mathbf{0 . 7 4 5}$ & $\mathbf{0 . 6 3 7}$ \\
Russia & $\mathbf{0 . 9 5 1}$ & $\mathbf{0 . 9 5 4}$ & $\mathbf{0 . 9 3 7}$ & $\mathbf{0 . 9 3 0}$ & $\mathbf{0 . 9 2 3}$ & $\mathbf{0 . 9 1 5}$ & $\mathbf{0 . 8 8 0}$ \\
Singapore & $\mathbf{0 . 9 5 6}$ & $\mathbf{0 . 9 5 1}$ & $\mathbf{0 . 9 6 4}$ & $\mathbf{0 . 9 7 5}$ & $\mathbf{0 . 9 9 3}$ & $\mathbf{0 . 9 9 1}$ & $\mathbf{0 . 8 8 6}$ \\
S. Africa & $\mathbf{0 . 9 9 5}$ & 1.033 & 1.079 & 1.106 & 1.139 & 1.170 & $\mathbf{0 . 9 6 7}$ \\
Sweden & 1.029 & 1.045 & 1.056 & 1.071 & 1.112 & 1.156 & 1.015 \\
Taiwan & 1.020 & $\mathbf{0 . 9 4 1}$ & $\mathbf{0 . 9 0 1}$ & $\mathbf{0 . 8 8 2}$ & $\mathbf{0 . 8 8 7}$ & $\mathbf{0 . 8 5 9}$ & $\mathbf{0 . 6 9 7}$ \\
UK & 1.010 & 1.057 & 1.102 & 1.133 & 1.174 & 1.219 & 1.388 \\
Japan & 1.002 & 1.018 & 1.043 & 1.089 & 1.114 & 1.151 & 1.617 \\
Swiss & $\mathbf{0 . 9 9 5}$ & $\mathbf{0 . 9 8 8}$ & $\mathbf{0 . 9 8 6}$ & $\mathbf{0 . 9 7 1}$ & $\mathbf{0 . 9 9 3}$ & 1.039 & $\mathbf{0 . 8 1 0}$ \\
Euro & $\mathbf{0 . 9 7 8}$ & $\mathbf{0 . 9 7 9}$ & $\mathbf{0 . 9 7 2}$ & $\mathbf{0 . 9 6 0}$ & $\mathbf{0 . 9 6 8}$ & $\mathbf{0 . 9 5 5}$ & $\mathbf{0 . 8 2 7}$ \\
\hline
\end{tabular}

